\title{
Antimicrobial susceptibility survey of pathogens isolated from selected patients in Northern Italy
} Elisabetta Maioli, Italo DeMandina, Simone Cagnacci, Clara Cassanelli, Fabrizio Cavallini,
Laura Gualco, Simona Roveta, Anna Marchese, Eugenio A. Debbia

Sezione di Microbiologia - DISCAT, Università di Genova

Key words: in- and out-patients, antimicrobial susceptibility patterns, Northern Italy

\section{ABSTRACT}

The Clinical Microbiology Laboratory of the University of Genoa participated, during the year 2003, in an international antimicrobial surveillance program. The collection of isolates was done according to the site of infection and/or type of patient. Four hundred twenty (420) clinical isolates were analyzed during this year and the frequencies of the different pathogens were investigated. A reference centre carried out susceptibility tests. Oxacillin-resistant Staphylococcus aureus represented $47.6 \%$ of all S. aureus isolates from blood stream infections and $33.3 \%$ of all S. aureus isolated from skin and soft tissue infections in hospitalised patients. These strains showed resistance to most of the antimicrobial agents evaluated, except vancomycin, teicoplanin, quinupristin/dalfopristin and linezolid which registered $100 \%$ of susceptibility. Some isolates from blood stream infections such as E. coli demonstrated resistance to ciprofloxacin (23.3\%), levofloxacin (20\%), and gatifloxacin (I6.6\%), and Klebsiella pneumoniae was resistant (18\%) to all fluoroquinolones tested. Pseudomonas aeruginosa manifested resistance to ciprofloxacin (16.6\%), while $27.7 \%$ of these strains were resistant both to levofloxacin and gatifloxacin. All the Enterobacter cloacae isolated from blood were susceptible to ciprofloxacin, levofloxacin and gatifloxacin. Haemophilus influenzae and Moraxella catarrhalis collected from community-acquired respiratory tract infections were all inhibited by ciprofloxacin, levofloxacin and gatifloxacin. E. coli isolated from urinary tract infections in hospitalised patients were resistant to ciprofloxacin, levofloxacin and gatifloxacin (2.7\%). All Salmonella spp. collected from samples of patients affected by infections of the gastro-intestinal tract were susceptible to all fluoroquinolones. Penicillin resistance in Streptococcus pneumoniae was found in $21.4 \%$ of isolates from patients with respiratory tract infections. Fluoroquinolone resistance was very rare among pneumococci except for ciprofloxacin. High rates of resistance to this drug (100\%); resistance to levofloxacin and gatifloxacin was detected only in $3.6 \%$ of cases while resistance to moxifloxacina was detected in $1.8 \%$ of cases. Macrolide resistance rates were around $35 \%$ to $41 \%$, tetracycline and trimethoprim/sulfamethoxazole resistance rates were relatively elevated (37.5\% and $32.1 \%$ resistance respectively).

\section{INTRODUCTION}

The importance of antimicrobial resistance among nosocomial and community-acquired pathogens is acknowledged worldwide, welldesigned antimicrobial surveillance programs are therefore of paramount importance in the fight against these microorganisms $(1,3,4,6)$.

These programs are also necessary to provide information on the trend in microbial occurrence in different geographical regions, to identify changes in the spectrum of microbial pathogens causing serious infection and to monitor trends in antimicrobial resistance patterns in nosocomial and community-acquired infections $(10,11,13$, 14) The information obtained from surveillance efforts have the potential to guide therapeutic approaches for serious infections and may have value in the prevention and control of infection and also to defining appropriate control measures for antimicrobial-resistant pathogens $(2,7,8,12)$. This information has been provided in recent years by several national and international antimicrobial surveillance program (1, 3-5). The
Laboratory of Clinical Microbiology of the University of Genoa was included in one of these international surveys designed to identify the predominant pathogens and antimicrobial resistance for both nosocomial and community-acquired infections, collecting consecutive isolates from clinically documented infections. The isolates are gathered according to the type of infection and susceptibility tests are carried out in a Central Microbiology Laboratory by reference broth microdilution methods according to NCCLS guidelines (9).

This study reports the antimicrobial susceptibility patterns of a collection of pathogens isolated from in- and out-patients affected by selected infections in Northern Italy.

\section{MATERIAL AND METHODS}

\section{Bacterial strains}

The collection of isolates was done according to the site of infection and/or type of patient. The contribution of this Laboratory (organism identification, date of isolation, antimicrobial suscepti- 
bility profile, etc.) included the first 20 consecutive episodes of blood stream infections (BSI) per month from January 2003 to December 2003, 94 consecutive episodes of community-acquired respiratory tract infections (CARTI), 50 consecutive isolates from skin and soft tissue infection (SSTI), 50 consecutive isolates from urinary-tract infections (UTI), 11 consecutive isolates of organisms considered producers of diarrheal diseases (gastroenteritis, GE) and 7 vancomycin-resistant enterococci (VRE) collected during the period January 2003 - December 2003. Just one isolate per patient was included in the study and all isolates were saved on transport swabs and sent to the monitoring center (Iowa, USA) for storage and further characterization by reference identification and susceptibility testing methods.

\section{SUSCEPTIBILITY TESTING}

All isolates were tested against antimicrobials by the broth microdilution method according to National Committee for Clinical Laboratory Standards (NCCLS) by the reference laboratory (Iowa, USA); breakpoints for resistance were those recommended by the NCCLS (9). Antimicrobial agents were obtained from their respective manufactures as laboratory grade powder and included penicillins (ampicillin, penicillin, amoxicillin, and oxacillin), b-lactamase inhibitor combinations (amoxicillin-clavulanate, ticarcillin-clavulanate, and piperacillin-tazobactam), cephalosporins (cefepime, cefuroxime, cefotaxime, ceftriaxone, ceftazidime, cefazolin, cefoxitin, cefaclor, and cefixime), carbapenems (imipenem and meropenem), a monobactam (aztreonam), fluoroquinolones (ciprofloxacin, levofloxacin, gatifloxacin, and garenoxacin [formerly BMS 284756]), aminoglycosides (amikacin, gentamicin, and tobramycin), macrolides (erythromycin, azithromycin, and clarithromycin), the oxazolidinone linezolid, the streptogramin quinupristin-dalfopristin, glycopeptides (vancomycin and teicoplanin), and other drugs, such as clindamycin, chloramphenicol, tetracycline, rifampin, and trimethoprim-sulfamethoxazole.

\section{RESULTS AND DISCUSSION}

Fourhundredtwenty (420) clinical isolates were analyzed during 2003 and the frequencies of the different pathogens were investigated. The collection and the distribution of the bacterial strain is reported in Table 1. Among these isolates, 223 were gram-negative, while 197 were gram-positive bacteria. Staphylococcus aureus (18.1\%) was the most frequent pathogen found with different incidence Escherichia coli (16.7\%),
Streptococcus pneumoniae (13.6\%), Enterococcus faecalis (6.4\%), Haemophilus influenzae (5.5\%) and Pseudomonas aeruginosa $(4.8 \%)$.

Table I - Distribution of the 420 isolates studied

\begin{tabular}{llll}
\hline BSI (2 10) & PATHOGEN & \multicolumn{2}{l}{ N. of isolates \% } \\
\hline & S. aureus & 42 & 20 \\
\hline & E. coli & 30 & 14.3 \\
\hline & E. faecalis & 22 & 10.5 \\
\hline & P. aeruginosa & 18 & 8.6 \\
\hline & E. cloacae & 14 & 6.7 \\
\hline & K. pneumoniae & 11 & 5.2 \\
\hline & Other' & 73 & 34.7 \\
\hline CARTI (94) & S. pneumoniae & 56 & 59.5 \\
\hline & H. influenzae & 22 & 23.4 \\
\hline & M. catarrhalis & 16 & 17.1 \\
\hline SSTI (50) & S. aureus & 33 & 66 \\
\hline & S. epidermidis & 13 & 26 \\
\hline & Other & \\
\hline UTI (48) & E. coli & 4 & 8 \\
\hline & Other & 37 & 77 \\
\hline GE (II) & Salmonella spp. & 1 I & 23 \\
\hline & E. coli & 3 & 63.6 \\
\hline & Aeromonas spp. & 1 & 27.3 \\
\hline VRE (7) & E. faecium & 4 & 5.1 \\
\hline & E. faecalis & 2 & 28.5 \\
\hline & E. avium & 1 & 14.5 \\
\hline
\end{tabular}

('Including S. maltophilia (8), S. epidermidis (5), P. mirabilis (5), K. oxytoca (4), A. baumannii (3). Corynebacterium spp. (3), S. group A (3), L. monocytogenes (3), M. morganii (2), S. liquefaciens (2), S. marcescens (2), S. coagulase neg. (2), S. haemolyticus (2), E. avium (2), S. mitis (2), Aeromonas spp (I), Bacillus spp. (I), B. cepacia (I), C. freundii (I), E. durans (I), S. anginosus (I), S. group B (I), S. pneumoniae (I), Streptococcus spp. (I), E. gallinarum (I), Enterococcus spp. (I), H. influenzae (I), S. paucimobilis (I), Staphilococcus spp. (I);

${ }^{2}$ Including E. faecalis (3), E. faecium (I);

${ }^{3}$ Including E. cloacae (2), P. aeruginosa (2), S. epidermidis (2), Enterococcus spp. (I), K. pneumoniae (I), Klebsiella spp. (I), S. aureus (I), S. saprophiticus (I))

Tables from 2 to 7 reported the in vitro activity of the antibiotic tested against the pathogens isolated from the different anatomic sites. Briefly, with respect to strains collected from blood stream infections. S. aureus (Table 2) resulted resistant in about $50 \%$ of the cases, including oxacillin, against the antibiotics tested with the exception of glycopeptides, linezolid, quinupristin/dalfopristin, chloramphenicol, tetracycline rifampicin, co-trimoxeazole, and nitrofurantoin which were found active against more than $90 \%$ of the bacterial strains. E. faecalis (Table 2) was inhibited by ampicillin, penicillin, linezolid, glycopeptides and nitrofurantoin, while the other compounds were active against about $50 \%$ of these strains. $E$. coli (Table 2) resulted susceptible to $\beta$-lactamaseresistant antibiotics in more than $70 \%$ of the strains. Fluoroquinolones showed activity against more than $75 \%$ of the cases while gentamycin 
inhibited all isolates. K. pneumoniae (Table 2) manifested susceptibility to the great majority of the $\beta$-lactam drugs, aminoglycosides, and to a lesser extend to other antibiotics. E. cloacae (Table 2) was found susceptible to cefepime, carbapenems, fluoroquinolones and amikacin, the other molecules were less active against this pathogen. P. aeruginosa (Table 2) as expected was resistant in about $40 \%$ of the cases, meropenem was fully inhibitory and ciprofloxacin was active on $83.4 \%$ of the strains tested.

Penicillin resistance in Streptococcus pneumoniae was found in $21.4 \%$ of isolates from patients with respiratory tract infections (Table 3 ).

Fluoroquinolone resistance was very rare among pneumococci except for ciprofloxacin, in fact pneumococci showed very high rates of resistance to this drug $(100 \%)$; resistance to levofloxacin and gatifloxacin was detected only in $3.6 \%$ of cases while resistance to moxifloxacin was detected in $1.8 \%$ of cases. Macrolide resistance rates were around $35 \%$ to $41 \%$, tetracycline and trimethoprim/sulfamethoxazole resistance rates were relatively elevated $(37.5 \%$ and $32.1 \%$ resistance respectively). The other respiratory pathogens, $H$. influenzae and $\mathrm{M}$. catarrhalis demonstrated susceptibility to all antibiotics tested with the exception of $H$. influenzae that was inhibited $82 \%$ by ampicillin (Table 3).

$S$. aureus isolated from soft tissue infections (Table 4) resulted resistant to oxacillin in the $33.3 \%$ of the strains. This level of unsuceptibility was found against many other antibiotics. Non B-lactam drugs with the exception of macrolides showed satisfactory in vitro activity against this pathogen. Similar results were registered with $S$. epidermidis with the exception of tetracycline which demonstrated activity on about $50 \%$ of the isolated. E. coli collected from UTI (Table 5) was in general more widely susceptible to all the antibiotics tested with the exception of cefazolin, tetracycline and trimethoprim/sulfamethoxazole . Salmonella spp. found in gastroenterical tract (Table 6) was in general inhibited by all the drugs. Low percentage of susceptible strains were detected when penicillins were tested.

Finally vancomycin-resistant enterococci (Table 7) resulted poorly susceptible to the drugs tested with the exception of linezolid and chloramphenicol which inhibited all strains.

The present findings indicate a large diffusion of antibiotic resistant strains in nosocomial settings however susceptibility tests also show a significant percentage of pathogens that are inhibited by various antibiotics.

Thus continuous national and international surveillance programs can provides accurate and timely data on the incidence of resistance in the major nosocomial pathogens This information must reach the clinicians (publication in national and international journals, Congresses, Meetings, Discussions, etc) in order to rationally influence their prescription habits and improve the outcome of therapy $(3-5,10,11)$.

Table 2. In vitro activity of the drugs against bacterial strains collected from blood stream infection

\begin{tabular}{|c|c|c|c|c|c|}
\hline $\begin{array}{c}\text { ORGANISM } \\
\text { (NO. TESTED) }\end{array}$ & ANTIBIOTIC & RANGE & MIC-50 & MIC-90 & $\begin{array}{c}\% \\
\text { SUSCEPTIBILITY }\end{array}$ \\
\hline \multirow{17}{*}{$\begin{array}{c}\text { E. faecalis } \\
\text { (22) }\end{array}$} & Ampicillin & $<=1-4$ & 2 & 4 & 100 \\
\hline & Penicillin & $2-16$ & 4 & 8 & 90 \\
\hline & Imipenem & $1-8$ & 1 & 8 & - \\
\hline & Meropenem & $4->16$ & 8 & $>16$ & - \\
\hline & Ciprofloxacin & $0,5->4$ & $>4$ & $>4$ & 51 \\
\hline & Levofloxacina & $1->4$ & $>4$ & $>4$ & 45.5 \\
\hline & Gatifloxacin & $0,25->4$ & 4 & $>4$ & 45.4 \\
\hline & Moxifloxacin & $0,25->4$ & 4 & $>4$ & - \\
\hline & Erythromycin & $0,5->8$ & $>8$ & $>8$ & 19 \\
\hline & Linezolid & $1-2$ & 1 & 2 & 100 \\
\hline & Quinupristin/Dalfopristin & $>=2$ & $>2$ & $>2$ & 0 \\
\hline & Vancomycin & $0,5->16$ & 1 & 2 & 90 \\
\hline & Teicoplanin & $<=2->16$ & $<=2$ & $<=2$ & 90 \\
\hline & Chloramphenicol & $4->16$ & $>16$ & $>16$ & 46 \\
\hline & Tetracycline & $<=2->8$ & $>8$ & $>8$ & 9 \\
\hline & Rifampin & $1->2$ & 2 & $>2$ & 13.7 \\
\hline & Nitrofurantoin & $<=0,16->32$ & $<=16$ & $<=16$ & 95.5 \\
\hline
\end{tabular}


Table 2. Continued

\begin{tabular}{|c|c|c|c|c|c|}
\hline $\begin{array}{c}\text { ORGANISM } \\
\text { (NO. TESTED) }\end{array}$ & ANTIBIOTIC & RANGE & MIC-50 & MIC-90 & $\begin{array}{c}\% \\
\text { SUSCEPTIBILITY }\end{array}$ \\
\hline \multirow[t]{27}{*}{ S.aureus (42) } & Ampicillin & $<=1->16$ & 8 & $>16$ & 39 \\
\hline & Penicillin & $<=0,016>32$ & 8 & $>32$ & 21.5 \\
\hline & Oxacillin & $<=0,25->2$ & 1 & $>2$ & 52.4 \\
\hline & Amoxicillin/Clavulanic acid & $<=1->16$ & $<=1$ & $>16$ & 52.4 \\
\hline & Piperacillin/Tazobactam & $0,5->256$ & 2 & 256 & 45.3 \\
\hline & Cefepime & $1->16$ & 4 & $>16$ & 52.4 \\
\hline & Ceftriaxone & $0,5->32$ & 8 & $>32$ & 54.8 \\
\hline & Ceftazidime & $4->16$ & 16 & $>16$ & 40.5 \\
\hline & Imipenem & $<=0,12->8$ & 0,25 & $>8$ & 57.2 \\
\hline & Meropenem & $0,06->16$ & 0,25 & $>16$ & 57.2 \\
\hline & Ertapenem & $<=0,06->8$ & 0,25 & $>8$ & 52.4 \\
\hline & Ciprofloxacin & $0,12->4$ & 4 & $>4$ & 47.7 \\
\hline & Levofloxacin & $<=0,03->4$ & 4 & $>4$ & 47.7 \\
\hline & Gatifloxacin & $<=0,03->4$ & 1 & 4 & 73.9 \\
\hline & Moxifloxacin & $<=0,03->4$ & 1 & 4 & - \\
\hline & Gentamicin & $<=2->8$ & $<=2$ & $>8$ & 52.4 \\
\hline & Erythromycin & $<=0,06->8$ & $>8$ & $>8$ & 47.7 \\
\hline & Clindamycin & $<=0,06->8$ & 0,12 & $>8$ & 59.6 \\
\hline & Linezolid & $0,5-2$ & 2 & 2 & 100 \\
\hline & Quinupristin/Dalfopristin & $<=0,25-1$ & 0,5 & 1 & 100 \\
\hline & Vancomycin & $0,5-2$ & 1 & 1 & 100 \\
\hline & Teicoplanin & $<=2-4$ & $<=2$ & $<=2$ & 100 \\
\hline & Chloramphenicol & $4->16$ & 8 & 8 & 90.5 \\
\hline & Tetracycline & $<=2$ & $<=2$ & $<=2$ & 100 \\
\hline & Rifampin & $<=0,25$ & $<=0,25$ & $<=0,25$ & 100 \\
\hline & Trimethoprim/Sulfamethoxazole & $<=0,5->2$ & $<=0,5$ & $<=0,5$ & 95.3 \\
\hline & Nitrofurantoin & $<=16->32$ & $<=16$ & $<=16$ & 96.7 \\
\hline
\end{tabular}

\begin{tabular}{|c|c|c|c|c|c|}
\hline $\begin{array}{l}\text { ORGANISM } \\
\text { (NO. TESTED) }\end{array}$ & ANTIBIOTIC & RANGE & MIC-50 & MIC-90 & $\begin{array}{c}\% \\
\text { SUSCEPTIBILITY }\end{array}$ \\
\hline \multirow[t]{28}{*}{ E. coli $(30)$} & Ampicillin & $2->16$ & $>16$ & $>16$ & 36.7 \\
\hline & Piperacillin & $<=1->128$ & 32 & $>128$ & 46.7 \\
\hline & Ticarcillin & $<=16-128$ & $>128$ & $>128$ & 36.7 \\
\hline & Amoxicillin/Clavulanic acid & $4->16$ & 8 & 16 & 70 \\
\hline & Ampicillin/Sulbactam & $2->32$ & 16 & 32 & 40 \\
\hline & Piperacillin/Tazobactam & $0,5-8$ & 2 & 2 & 100 \\
\hline & Ticarcillin/Clavulanic acid & $<=16-128$ & $<=16$ & 64 & 73.4 \\
\hline & Cefepime & $<=0,12-0,5$ & $<=0,12$ & 0,25 & 100 \\
\hline & Cefuroxime & $2->16$ & 4 & 8 & 70 \\
\hline & Ceftriaxone & $<=0,25$ & $<=0,25$ & $<=0,25$ & 100 \\
\hline & Ceftazidime & $<=1-16$ & $<=1$ & $<=1$ & 93.4 \\
\hline & Cefazolin & $<=2->16$ & $<=2$ & $>16$ & 86.7 \\
\hline & Cefoxitin & $2-32$ & 4 & 8 & 90 \\
\hline & Imipenem & $<=0,12-0,25$ & 0,25 & 0,25 & 100 \\
\hline & Meropenem & $<=0,008-0,03$ & 0,016 & 0,03 & 100 \\
\hline & Ertapenem & $<=0,06$ & $<=0,06$ & $<=0,06$ & 100 \\
\hline & Aztreonam & $<=0,12-16$ & $<=0,12$ & 0,25 & 96.7 \\
\hline & NalidixicAcid & $1->32$ & 2 & $>32$ & 70 \\
\hline & Ciprofloxacin & $<=0,03->4$ & $<=0,03$ & $>4$ & 76.7 \\
\hline & Levofloxacina & $<=0,03->4$ & $<=0,03$ & 4 & 80 \\
\hline & Gatifloxacin & $<=0,03->4$ & $<=0,03$ & $>4$ & 83.4 \\
\hline & Moxifloxacin & $<=0,03->4$ & 0,06 & $>4$ & - \\
\hline & Amikacin & $<0,25-8$ & 2 & 4 & 100 \\
\hline & Gentamicin & $<=2->8$ & $<=2$ & $<=2$ & 93.4 \\
\hline & Tobramycin & $0,25-8$ & 0,5 & 2 & 93.4 \\
\hline & Tetracycline & $<=2->8$ & $>8$ & $>8$ & 40 \\
\hline & Trimethoprim/Sulfamethoxazole & $<=0,5->2$ & $<=0,5$ & $>2$ & 66.7 \\
\hline & Nitrofurantoin & $<=16->32$ & $<=16$ & 32 & 93.4 \\
\hline
\end{tabular}


Table 2. Continued

\begin{tabular}{|c|c|c|c|c|c|}
\hline $\begin{array}{l}\text { ORGANISM } \\
\text { (NO. TESTED) }\end{array}$ & ANTIBIOTIC & RANGE & MIC-50 & MIC-90 & $\begin{array}{c}\% \\
\text { SUSCEPTIBILITY }\end{array}$ \\
\hline \multirow[t]{15}{*}{ P. aeruginosa (18) } & Piperacillin & $4->128$ & 8 & $>128$ & 66.7 \\
\hline & Ticarcillin & $<=16->128$ & 64 & $>128$ & 61.2 \\
\hline & Piperacillin/Tazobactam & $4-256$ & 8 & 256 & 72.3 \\
\hline & Ticarcillin/Clavulanic acid & $<=16->128$ & 64 & $>128$ & 55.6 \\
\hline & Cefepime & $1>16$ & 2 & $>16$ & 76.7 \\
\hline & Ceftazidime & $2->16$ & 4 & $>16$ & 66.7 \\
\hline & Imipenem & $0,5->8$ & 1 & $>8$ & 72.3 \\
\hline & Meropenem & $0,12-16$ & 1 & 4 & 100 \\
\hline & Aztreonam & $4->16$ & 8 & $>16$ & 50 \\
\hline & Ciprofloxacin & $0,12->4$ & 0,25 & $>4$ & 83.4 \\
\hline & Levofloxacina & $0,25->4$ & 1 & $>4$ & 72.3 \\
\hline & Gatifloxacin & $0,25->4$ & 1 & $>4$ & 72.3 \\
\hline & Moxifloxacin & $0,5->4$ & 4 & $>4$ & - \\
\hline & Amikacin & $2-32$ & 4 & 32 & 89 \\
\hline & Tobramycin & $0,25->16$ & 0,5 & $>16$ & 83.4 \\
\hline
\end{tabular}

\begin{tabular}{|c|c|c|c|c|c|}
\hline $\begin{array}{l}\text { ORGANISM } \\
\text { (NO. TESTED) }\end{array}$ & ANTIBIOTIC & RANGE & MIC-50 & MIC-90 & $\begin{array}{c}\% \\
\text { SUSCEPTIBILITY }\end{array}$ \\
\hline \multirow{28}{*}{$\begin{array}{c}\text { E. cloacae } \\
(14)\end{array}$} & Ampicillin & $>16$ & $>16$ & $>16$ & 100 \\
\hline & Piperacillin & $<=1->128$ & 8 & $>128$ & 57 \\
\hline & Ticarcillin & $<=16->128$ & $<=16$ & $>128$ & 50 \\
\hline & Amoxicillin/Clavulanic acid & $>16$ & $>16$ & $>16$ & 0 \\
\hline & Ampicillin/Sulbactam & $0,5->32$ & 32 & $>32$ & 28,5 \\
\hline & Piperacillin/Tazobactam & $1-256$ & 4 & 128 & 64 \\
\hline & Ticarcillin/Clavulanic acid & $<=16->128$ & $<=16$ & $>128$ & 57 \\
\hline & Cefepime & $<=0,12-16$ & $<=0,12$ & 8 & 100 \\
\hline & Cefuroxime & $8->16$ & 16 & $>16$ & 29 \\
\hline & Ceftriaxone & $<=0,25->32$ & 0,5 & $>32$ & - \\
\hline & Ceftazidime & $<=1->16$ & $<=1$ & $>16$ & 57 \\
\hline & Cefazolin & $>16$ & $>16$ & $>16$ & 0 \\
\hline & Cefoxitin & $>32$ & $>32$ & $>32$ & 0 \\
\hline & Imipenem & $<=0,12-0,25$ & 0.25 & 0.25 & 100 \\
\hline & Meropenem & $0,03-0,12$ & 0,03 & 0,12 & 100 \\
\hline & Ertapenem & $<=0,06-2$ & 0,12 & 2 & 100 \\
\hline & \begin{tabular}{|l|} 
Aztreonam \\
\end{tabular} & $<=0,12->16$ & 0,25 & $>16$ & 57 \\
\hline & Nalidixic Acid & $2->32$ & 4 & 16 & 86 \\
\hline & Ciprofloxacin & $<=0,03-0,5$ & $<=0,03$ & 0,5 & 100 \\
\hline & Levofloxacina & $<=0,03-0,5$ & $<=0,03$ & 0,5 & 100 \\
\hline & Gatifloxacin & $<=0,03-0,5$ & 0,06 & 0,25 & 100 \\
\hline & Moxifloxacin & $0,06-1$ & 1 & 1 & - \\
\hline & Amikacin & $1-8$ & 1 & 2 & 100 \\
\hline & Gentamicin & $<=2->8$ & $<=2$ & 8 & - \\
\hline & Tobramycin & $0,25->16$ & 0,5 & 16 & 79 \\
\hline & Tetracycline & $<=2->8$ & 4 & $>8$ & 72 \\
\hline & Nitrofurantoin & $>=32$ & $>32$ & $>32$ & 36 \\
\hline & Trimethoprim/Sulfamethoxazole & $<=0,5->2$ & $<0,5$ & $<0,5$ & 93 \\
\hline
\end{tabular}


Table 2. Continued

\begin{tabular}{|c|c|c|c|c|c|}
\hline $\begin{array}{l}\text { ORGANISM } \\
\text { (NO. TESTED) }\end{array}$ & ANTIBIOTIC & RANGE & MIC-50 & MIC-90 & $\begin{array}{c}\% \\
\text { SUSCEPTIBILITY }\end{array}$ \\
\hline \multirow{27}{*}{$\begin{array}{c}\text { K. pneumoniae } \\
\text { (11) }\end{array}$} & Ampicillin & $>=16$ & $>16$ & $>16$ & 0 \\
\hline & Piperacillin & $2->128$ & 16 & $>128$ & 54.6 \\
\hline & Ticarcillin & $<=16->128$ & $>128$ & $>128$ & 9 \\
\hline & Ampicillin/Sulbactam & $4-32$ & 8 & 32 & 36.4 \\
\hline & Piperacillin/Tazobactam & $1-16$ & 2 & 16 & 100 \\
\hline & Ticarcillin/Clavulanic acid & $<=16-128$ & $<=16$ & 64 & 82 \\
\hline & Cefepime & $<=0,12$ & $<=0,12$ & 2 & 100 \\
\hline & Cefuroxime & $1->16$ & 4 & $>16$ & 54.6 \\
\hline & Ceftriaxone & $<=0,25->32$ & $<=0.25$ & 32 & 82 \\
\hline & Ceftazidime & $<=1->16$ & $<=1$ & $>16$ & 82 \\
\hline & Cefazolin & $<=2->16$ & $<=2$ & $>16$ & 82 \\
\hline & Cefoxitin & $1-8$ & 4 & 8 & 100 \\
\hline & Imipenem & $<=0,12-0,25$ & 0,25 & 0,25 & 100 \\
\hline & Meropenem & 0,03 & 0,03 & 0,03 & 100 \\
\hline & Ertapenem & $<=0,06$ & $<=0,06$ & $<=0,06$ & 100 \\
\hline & Aztreonam & $<=0.12->16$ & $<=0.12$ & $>16$ & 82 \\
\hline & NalidixicAcid & $2->32$ & 2 & $>32$ & 82 \\
\hline & Ciprofloxacin & $<=0,03->4$ & $<=0,03$ & 4 & 82 \\
\hline & Levofloxacina & $<=0,03->4$ & 0,06 & - & 18 \\
\hline & Gatifloxacin & $<=0,03->4$ & 0,06 & $>4$ & 82 \\
\hline & Moxifloxacin & $<=0,03->4$ & 0,012 & 4 & - \\
\hline & Amikacin & $1-2$ & 2 & 2 & 100 \\
\hline & GentamicinLow & $<=2$ & $<=2$ & $<=2$ & 100 \\
\hline & Tobramycin & $0,25-4$ & 0,5 & 1 & 100 \\
\hline & Tetracycline & $<=2->8$ & $<=2$ & $>8$ & 72.7 \\
\hline & Trimethoprim/Sulfamethoxazole & $<=0,5->2$ & $<=0,5$ & 1 & 90 \\
\hline & Nitrofurantoin & $<016->32$ & 32 & $>32$ & 66.7 \\
\hline
\end{tabular}

Table 3. In vitro activity of the drugs against respiratory pathogens

\begin{tabular}{|c|c|c|c|c|c|}
\hline $\begin{array}{l}\text { ORGANISM } \\
\text { (NO. TESTED) }\end{array}$ & ANTIBIOTIC & RANGE & MIC-50 & MIC-90 & $\begin{array}{c}\% \\
\text { SUSCEPTIBILITY }\end{array}$ \\
\hline \multirow{22}{*}{$\begin{array}{c}\text { S. pneumoniae } \\
(56)\end{array}$} & Ampicillin & $<=0,5-4$ & $<=0,5$ & $<=0,5$ & - \\
\hline & Penicillin & $<=0,03-4$ & $<=0,03$ & 0,25 & 78.6 \\
\hline & Amoxicillin/Clavulanic acid & $<=0,06-8$ & $<=0,06$ & $<=0,06$ & 98.2 \\
\hline & Azithromycin & $<=0,5->4$ & $<=0,5$ & $>4$ & 64.3 \\
\hline & Cefepime & $<=0,06-4$ & $<=0,06$ & 0,5 & 96.4 \\
\hline & Cefuroxime & $<=0,06->8$ & $<=0,06$ & 2 & 89.3 \\
\hline & Ceftriaxone & $<=0,008-8$ & 0,03 & 0,5 & 96.5 \\
\hline & Cefpodoxime & $<=0,03->4$ & $<=0,03$ & 1 & 89.3 \\
\hline & Cefprozil & $<=0,12->16$ & $<=0,12$ & 1 & 92.9 \\
\hline & Ciprofloxacin & $0,5->16$ & 1 & 2 & 0 \\
\hline & Levofloxacina & $0,5->4$ & 1 & 1 & 96.4 \\
\hline & Gatifloxacin & $0,12->4$ & 0,25 & 0,5 & 96.4 \\
\hline & Moxifloxacin & $0,06-4$ & 0,12 & 0,25 & 98.2 \\
\hline & Erythromycin & $<=0,25->32$ & $<=0,25$ & $>32$ & 59 \\
\hline & Clarithromycin & $<=0,25->32$ & $<=0,25$ & $>32$ & 59 \\
\hline & Clindamycin & $<=0,25->2$ & $<=0,25$ & $>2$ & 69.7 \\
\hline & Quinupristin/Dalfopristin & $<=0,5-1$ & $<=0,5$ & $<=0,5$ & 100 \\
\hline & Vancomycin & $<=0,06-0,5$ & 0,25 & 0,5 & 100 \\
\hline & Chloramphenicol & $<=2-16$ & $<=2$ & 16 & 85.8 \\
\hline & Tetracycline & $<=2->16$ & $<=2$ & $>16$ & 62.5 \\
\hline & Rifampin & $<=0,5$ & $<=0,5$ & $<=0,5$ & 100 \\
\hline & Trimethoprim/Sulfamethoxazole & $<=2->4$ & $<=2$ & $>16$ & 68 \\
\hline
\end{tabular}


Table 3. Continued

\begin{tabular}{|c|c|c|c|c|c|}
\hline $\begin{array}{c}\text { ORGANISM } \\
\text { (NO. TESTED) }\end{array}$ & ANTIBIOTIC & RANGE & MIC-50 & MIC-90 & $\begin{array}{c}\% \\
\text { SUSCEPTIBILITY }\end{array}$ \\
\hline \multirow{24}{*}{$\begin{array}{c}\text { H. influenzae } \\
(22)\end{array}$} & Amoxicillin/Clavulanic acid & $0,25-1$ & 0,25 & 0,5 & 100 \\
\hline & Ampicillin & $<=0,5->4$ & $<=0,5$ & $>4$ & 82 \\
\hline & Azithromycin & $<=0,5-2$ & 1 & 1 & 100 \\
\hline & Cefepime & $<=0,06-0,12$ & $<=0,06$ & $<=0,06$ & 100 \\
\hline & Cefpodoxime & $<=0,03-0,06$ & $<=0,03$ & 0,06 & 100 \\
\hline & Cefprozil & $0,5-4$ & 1 & 4 & 100 \\
\hline & Ceftriaxone & $<=0,08$ & $<=0,08$ & $<=0,08$ & 100 \\
\hline & Cefuroxime & $0,25-1$ & 0,5 & 1 & 100 \\
\hline & Chloramphenicol & $<=2$ & $<=2$ & $<=2$ & 100 \\
\hline & Ciprofloxacin & $<=0,12$ & $<=0,12$ & $<=0,12$ & 100 \\
\hline & Clarithromycin & $1-16$ & 4 & 8 & 100 \\
\hline & Clindamycin & $1-8$ & 4 & 8 & - \\
\hline & Erythromycin & $2-8$ & 4 & 4 & - \\
\hline & Gatifloxacin & $<=0,03$ & $<=0,03$ & $<=0,03$ & 100 \\
\hline & Levofloxacina & $<=0,03$ & $<=0,03$ & $<=0,03$ & 100 \\
\hline & Meropenem & $0,03-0,16$ & 0,03 & 0,06 & 100 \\
\hline & Moxifloxacin & $<=0,03$ & $<=0,03$ & $<=0,03$ & 100 \\
\hline & Penicillin & $0,12->4$ & 0,25 & $>4$ & - \\
\hline & Piperacillin/Tazobactam & $<=0,12$ & $<=0,12$ & $<=0,12$ & 100 \\
\hline & Quinupristin/Dalfopristin & $2-4$ & 4 & 4 & - \\
\hline & Rifampin & $<=0,5$ & $<=0,5$ & $<=0,5$ & 100 \\
\hline & Tetracycline & $<=2$ & $<=2$ & $<=2$ & 100 \\
\hline & TrimSulfa & $<=0,5$ & $<=0,5$ & $<=0,5$ & 100 \\
\hline & Vancomycin & $>4$ & $>4$ & $>4$ & - \\
\hline
\end{tabular}

\begin{tabular}{|c|l|c|c|c|c|}
\hline $\begin{array}{c}\text { ORGANISM } \\
\text { (NO. TESTED) }\end{array}$ & \multicolumn{1}{|c|}{ ANTIBIOTIC } & RANGE & MIC-50 & MIC-90 & $\begin{array}{c}\text { \% } \\
\text { SUSCEPTIBILITY }\end{array}$ \\
\hline \multirow{4}{*}{ M. catarrhalis (16) } & Ampicillin & $<=0,5-2$ & 1 & 2 & - \\
\cline { 2 - 6 } & Penicillin & $<=0,03->4$ & 4 & $>4$ & - \\
\cline { 2 - 6 } & Amoxicillin/Clavulanic acid & $<=0,06-0,25$ & 0,12 & 0,25 & - \\
\cline { 2 - 6 } & Cefepime & $0,12-1$ & 0,5 & 1 & 100 \\
\cline { 2 - 6 } & Cefuroxime & $0,25-2$ & 1 & 2 & - \\
\cline { 2 - 6 } & Ceftriaxone & $<=0,08-0,5$ & 0,12 & 0,5 & 100 \\
\cline { 2 - 6 } & Cefpodoxime & $0,12-1$ & 0,5 & 0,5 & - \\
\cline { 2 - 6 } & Cefprozil & $0,5-2$ & 1 & 2 & - \\
\cline { 2 - 6 } & Ciprofloxacin & $<=0,12$ & $<=0,12$ & $<=0,12$ & 100 \\
\cline { 2 - 6 } & Levofloxacina & $<=0,03-0,06$ & $<=0,03$ & 0,06 & 100 \\
\cline { 2 - 6 } & Gatifloxacin & $<=0,03$ & $<=0,03$ & $<=0,03$ & 100 \\
\cline { 2 - 6 } & Moxifloxacin & $<=0,03-0,06$ & 0,06 & 0,06 & - \\
\cline { 2 - 6 } & Azithromycin & $<=0,5$ & $<=0,5$ & $<=0,5$ & - \\
\cline { 2 - 6 } & Chloramphenicol & $<=2$ & $<=2$ & $<=2$ & 100 \\
\cline { 2 - 6 } & Tetracycline & $<=2$ & $<=2$ & $<=2$ & 100 \\
\cline { 2 - 6 } & Rifampin & $<=0,5$ & $<=0,5$ & $<=0,5$ & - \\
\cline { 2 - 6 } & Trimethoprim/Sulfamethoxazole & $<=0,5$ & $<=0,5$ & $<=0,5$ & 100 \\
\hline
\end{tabular}


Table 4. In vitro activity of the drugs against bacterial strains collected from soft tissue infections

\begin{tabular}{|c|c|c|c|c|c|}
\hline $\begin{array}{c}\text { ORGANISM } \\
\text { (NO. TESTED) }\end{array}$ & ANTIBIOTIC & RANGE & MIC-50 & MIC-90 & $\begin{array}{c}\% \\
\text { SUSCEPTIBILITY }\end{array}$ \\
\hline \multirow[t]{26}{*}{ S.aureus (33) } & Ampicillin & $<=1->16$ & 16 & $>16$ & 9 \\
\hline & Penicillin & $0,06->32$ & 16 & $>32$ & 9 \\
\hline & Oxacillin & $<=0,25->2$ & 0,25 & $>2$ & 66.7 \\
\hline & Amoxicillin/Clavulanic acid & $<=1->16$ & $<=1$ & $>16$ & 66.7 \\
\hline & Cefepime & $2->16$ & 4 & $>16$ & 66.7 \\
\hline & Ceftriaxone & $4->32$ & 4 & $>32$ & 66.7 \\
\hline & Ceftazidime & $4->16$ & 8 & $>16$ & 54.6 \\
\hline & Imipenem & $<=0,5->8$ & $<=0,5$ & $>8$ & 72.7 \\
\hline & Meropenem & $0,12->16$ & 0,12 & $>16$ & 66.7 \\
\hline & Ertapenem & $<=0,06->8$ & 0,25 & $>8$ & 66.7 \\
\hline & Ciprofloxacin & $0,12->4$ & 0,5 & $>4$ & 72.7 \\
\hline & Levofloxacina & $0,12->4$ & 0,25 & 4 & 66.7 \\
\hline & Gatifloxacin & $<=0,03->4$ & 0,06 & 4 & 94 \\
\hline & Moxifloxacin & $<=0,03-4$ & $<=0,03$ & 2 & 78.8 \\
\hline & Gentamicin & $<=2->8$ & $<=2$ & $>8$ & 69.7 \\
\hline & Erythromycin & $0,12->8$ & 0,25 & $>8$ & 60.7 \\
\hline & Clindamycin & $<=0,06->8$ & 0,12 & $>8$ & 72.7 \\
\hline & Linezolid & $1-2$ & 2 & 2 & 100 \\
\hline & Quinupristin/Dalfopristin & $<=0,25-1$ & $<=0,25$ & 0,5 & 100 \\
\hline & Vancomycin & $0,5-2$ & 1 & 2 & 100 \\
\hline & Teicoplanin & $<=2-4$ & $<=2$ & $<=2$ & 100 \\
\hline & Chloramphenicol & $4->16$ & 8 & 8 & 97 \\
\hline & Tetracycline & $<=2->8$ & $<=2$ & $<=2$ & 91 \\
\hline & Rifampin & $<=0,25->2$ & $<=0,25$ & $>2$ & 88 \\
\hline & Trimethoprim/Sulfamethoxazole & $<=0,5-1$ & $<=0,5$ & $<=0,5$ & 97 \\
\hline & Nitrofurantoin & $<=16-32$ & $<=16$ & $<=16$ & 100 \\
\hline
\end{tabular}

\begin{tabular}{|c|c|c|c|c|c|}
\hline $\begin{array}{l}\text { ORGANISM } \\
\text { (NO. TESTED) }\end{array}$ & ANTIBIOTIC & RANGE & MIC-50 & MIC-90 & $\begin{array}{c}\% \\
\text { SUSCEPTIBILITY }\end{array}$ \\
\hline \multirow{27}{*}{$\begin{array}{c}\text { S. epidermidis } \\
\text { (13) }\end{array}$} & Ampicillin & $<=1->16$ & 2 & $>16$ & 38.5 \\
\hline & Penicillin & $0,25->32$ & 2 & 4 & 0 \\
\hline & Oxacillin & $<=0,25->2$ & $>2$ & $>2$ & 46.2 \\
\hline & Amoxicillin/Clavulanic acid & $<=1->16$ & $<=1$ & $>16$ & 84.7 \\
\hline & Piperacillin/Tazobactam & $<=0,25-32$ & 1 & 16 & 84.7 \\
\hline & Cefepime & $0,5->16$ & 4 & $>16$ & 84.7 \\
\hline & Ceftriaxone & $0,5->32$ & 16 & 32 & 54 \\
\hline & Ceftazidime & $4->16$ & 16 & $>16$ & 38.5 \\
\hline & Imipenem & $<=0,5->8$ & $<=0,5$ & 8 & 84.7 \\
\hline & Meropenem & $0,06-8$ & 1 & 8 & 69.3 \\
\hline & Ertapenem & $0,12->8$ & 2 & $>8$ & 69.3 \\
\hline & Ciprofloxacin & $0,12->4$ & $>4$ & $>4$ & 23 \\
\hline & Levofloxacina & $0,12->4$ & 4 & $>4$ & 30.8 \\
\hline & Gatifloxacin & $0,12-4$ & 2 & 2 & 92.4 \\
\hline & Moxifloxacin & $0,06-4$ & 2 & 2 & 30.7 \\
\hline & Gentamicin & $<=2->8$ & 4 & $>8$ & 54 \\
\hline & Erythromycin & $0,12->8$ & $>8$ & $>8$ & 38.5 \\
\hline & Clindamycin & $<=0,06->8$ & $<=0,06$ & $>8$ & 69.3 \\
\hline & Linezolid & $0,5-1$ & 1 & 1 & 100 \\
\hline & Quinupristin/Dalfopristin & $<=0,25$ & $<=0,25$ & $<=0,25$ & 100 \\
\hline & Vancomycin & $0,5-2$ & 1 & 2 & 100 \\
\hline & Teicoplanin & $<=2-16$ & $<=2$ & 8 & 92.3 \\
\hline & Chloramphenicol & $4->16$ & 4 & $>16$ & 84.7 \\
\hline & Tetracycline & $<=2->8$ & $<=2$ & $>8$ & 54 \\
\hline & Rifampin & $<=0,25->2$ & $<=0,25$ & 0,5 & 92.3 \\
\hline & Trimethoprim/Sulfamethoxazole & $<=0,5->2$ & $<=0,5$ & $>2$ & 61.6 \\
\hline & Nitrofurantoin & $<=16$ & $<=16$ & $<=16$ & 100 \\
\hline
\end{tabular}


Table 5. In vitro activity of the drugs against bacterial strains collected from urinary tract infections

\begin{tabular}{|c|c|c|c|c|c|}
\hline $\begin{array}{c}\text { ORGANISM } \\
\text { (NO. TESTED) }\end{array}$ & ANTIBIOTIC & RANGE & MIC-50 & MIC-90 & $\begin{array}{c}\% \\
\text { SUSCEPTIBILITY }\end{array}$ \\
\hline \multirow[t]{26}{*}{ E. coli $(37)$} & Ampicillin & $2->16$ & 4 & $>16$ & 56.8 \\
\hline & Piperacillin & $<=1->128$ & 2 & $>128$ & 59.5 \\
\hline & Amoxicillin/Clavulanic acid & $2->16$ & 8 & 16 & 81 \\
\hline & Ampicillin/Sulbactam & $1->32$ & 4 & $>32$ & 62.2 \\
\hline & Piperacillin/Tazobactam & $0,5-8$ & 2 & 4 & 100 \\
\hline & Cefepime & $<=0,12-2$ & $<=0,12$ & 0,25 & 100 \\
\hline & Cefuroxime & $0,5->16$ & 4 & 8 & 89.2 \\
\hline & Cefazolin & $<=2->16$ & $<=2$ & 4 & 1.9 \\
\hline & Cefoxitin & $<=0,25->32$ & 4 & 4 & 97.3 \\
\hline & Cefoperazone & $<=0,25->32$ & $<=0,25$ & 4 & 97.3 \\
\hline & Ceftazidime & $<=1->16$ & $<=1$ & $<=1$ & 97.3 \\
\hline & Ceftriaxone & $<=0,25-16$ & $<=0,25$ & $<=0,25$ & 97.3 \\
\hline & Cefoperazone/Sulbactam & $<=0,25->4$ & $<=0,25$ & 2 & 94.6 \\
\hline & Imipenem & $<=0,5$ & $<=0,5$ & $<=0,5$ & 100 \\
\hline & Meropenem & $<=0,008-0,03$ & 0,016 & 0,016 & 100 \\
\hline & Aztreonam & $<=0,12->16$ & $<=0,12$ & $<=0,12$ & 97.3 \\
\hline & Nalidixic Acid & $<=4->32$ & $<=4$ & $<=4$ & 94.6 \\
\hline & Ciprofloxacin & $<=0,03->4$ & $<=0,03$ & $<=0,03$ & 97.3 \\
\hline & Levofloxacin & $<=0,03->4$ & $<=0,03$ & 0,06 & 97.3 \\
\hline & Gatifloxacin & $<=0,03->4$ & $<=0,03$ & 0,06 & 97.3 \\
\hline & Moxifloxacin & $<=0,03->4$ & 0,06 & 0,06 & - \\
\hline & Amikacin & $0,5-4$ & 1 & 2 & 100 \\
\hline & Gentamicin & $<=2->8$ & $<=2$ & $<=2$ & 94.6 \\
\hline & Tetracycline & $<=2->8$ & $<=2$ & $>8$ & 32. \\
\hline & Trimethoprim/Sulfamethoxazole & $<=0,5->2$ & $<=0,5$ & 2 & 54 \\
\hline & Nitrofurantoin & $<=16->32$ & $<=16$ & $>32$ & 86.5 \\
\hline
\end{tabular}

Table 6. In vitro activity of the drugs against 7 Salmonella spp. Collected from gastrointestinal tract infections

\begin{tabular}{|c|c|c|c|c|c|}
\hline $\begin{array}{l}\text { ORGANISM } \\
\text { (NO. TESTED) }\end{array}$ & ANTIBIOTIC & RANGE & MIC-50 & MIC-90 & $\begin{array}{c}\% \\
\text { SUSCEPTIBILITY }\end{array}$ \\
\hline \multirow{23}{*}{$\begin{array}{l}\text { Salmonella } \\
\text { spp.(7) }\end{array}$} & Ampicillin & $<=1-16$ & 2 & 16 & 71.5 \\
\hline & Piperacillin & $<=1->128$ & 4 & $>128$ & 71.5 \\
\hline & Amoxicillin/Clavulanic acid & $0,5-4$ & 1 & 2 & 71.5 \\
\hline & Ampicillin/Sulbactam & $1->32$ & 1 & 32 & 71.5 \\
\hline & Piperacillin/Tazobactam & $<=0,5-8$ & 4 & 4 & 100 \\
\hline & Cefepime & $<=0,12-0,25$ & $<=0,12$ & $<=0,12$ & 100 \\
\hline & Cefuroxime & $4-16$ & 4 & 4 & 87.5 \\
\hline & Ceftriaxone & $<=0,25$ & $<=0,25$ & $<=0,25$ & 100 \\
\hline & Ceftazidime & $<=1$ & $<=1$ & $<=1$ & 100 \\
\hline & Cefazolin & $<=2-8$ & $<=2$ & 4 & 100 \\
\hline & Cefoxitin & $2-8$ & 2 & 2 & 100 \\
\hline & Cefoperazone & $<=0,25->32$ & 0,5 & 8 & 87.5 \\
\hline & Cefoperazone/Sulbactam & $<=0,25->16$ & 0,5 & 4 & - \\
\hline & Imipenem & $<=0,5$ & - & - & 100 \\
\hline & Aztreonam & $<=0,12-0,25$ & $<=0,12$ & $<=0,12$ & 100 \\
\hline & Nalidixic Acid & $<=4-8$ & $<=4$ & $<=4$ & 100 \\
\hline & Ciprofloxacin & $<=0,03$ & $<=0,03$ & $<=0,03$ & 100 \\
\hline & Levofloxacina & $<=0,03-0,06$ & 0,06 & 0,06 & 100 \\
\hline & Gatifloxacin & $<=0,03-0,06$ & $<=0,03$ & 0,06 & 100 \\
\hline & Moxifloxacin & $<=0,03-0,12$ & 0,06 & 0,12 & - \\
\hline & Gentamicin & $<=2$ & $<=2$ & $<=2$ & 100 \\
\hline & Tetracycline & $<=2->8$ & $<=2$ & $>8$ & 85.8 \\
\hline & Trimethoprim/Sulfamethoxazole & $<=0,5->2$ & $<=0,5$ & $<=0,5$ & 85.8 \\
\hline
\end{tabular}


Table 7. In vitro activity of the drugs against 7 Enterococcus spp. vancomycin-resistant

\begin{tabular}{|c|c|c|c|c|c|}
\hline $\begin{array}{l}\text { ORGANISM } \\
\text { (NO. TESTED) }\end{array}$ & ANTIBIOTIC & RANGE & MIC-50 & MIC-90 & $\begin{array}{c}\% \\
\text { SUSCEPTIBILITY }\end{array}$ \\
\hline \multirow{19}{*}{$\begin{array}{l}\text { Vancomycin- } \\
\text { resistant } \\
\text { Enterococcus } \\
\text { spp.(7) }\end{array}$} & Ampicillin & $2->16$ & $>16$ & $>16$ & 28.6 \\
\hline & Penicillin & $8->32$ & $>32$ & $>32$ & 28.6 \\
\hline & $\begin{array}{l}\text { Amoxicillin/Clavulanic } \\
\text { acid }\end{array}$ & $2 \rightarrow>16$ & $>16$ & $>16$ & - \\
\hline & Imipenem & $8->8$ & $>8$ & $>8$ & - \\
\hline & Ertapenem & $>8$ & $>8$ & $>8$ & - \\
\hline & Ciprofloxacin & $>4$ & $>4$ & $>4$ & 12.5 \\
\hline & Levofloxacin & $>4$ & $>4$ & $>4$ & 0 \\
\hline & Gatifloxacin & $>4$ & $>4$ & $>4$ & 0 \\
\hline & Moxifloxacin & $>4$ & $>4$ & $>4$ & - \\
\hline & Erythromycin & $>8$ & $>8$ & $>8$ & 0 \\
\hline & Clindamycin & $>8$ & $>8$ & $>8$ & - \\
\hline & Linezolid & $1-2$ & 2 & 2 & 100 \\
\hline & Quinupristin/Dalfopristin & $0,5->2$ & 1 & $>2$ & 71.4 \\
\hline & Vancomycin & $>16$ & $>16$ & $>16$ & 0 \\
\hline & Teicoplanin & $>16$ & $>16$ & $>16$ & 0 \\
\hline & Chloramphenicol & $4-8$ & 8 & 8 & 100 \\
\hline & Tetracycline & $<=2->8$ & $<=2$ & $>8$ & 85.7 \\
\hline & Rifampin & $2->2$ & $>2$ & $>2$ & 0 \\
\hline & Nitrofurantoin & $<=16->32$ & $>32$ & $>32$ & 43 \\
\hline
\end{tabular}

\section{REFERENCES}

1. Clark NM, Patterson J, Lynch JP. Antimicrobial resistance among Gram-negative organisms in the intensive care unit. Curr Op Crit Care 2003; 9: 413-23.

2. Cockerill FR, Smith TF. Response of the Clinical Microbiology laboratory to emerging (new) and reemerging infectious diseases. J Clin Microbiol 2004; 42: 2359-65.

3. Felmingham $\mathrm{D}$. The need for antimicrobial resistance surveillance. J Antimicrob Chemother 2002; 50, (suppl): 1-7.

4. Gastmeier P. Nosocomial infection surveillance and control policies. Curr Opin Infect Dis 2004; 17: 295-301.

5. Jones RN, Masterson R. Determining the value of antimicrobial surveillance programs. Diagn Microbiol Infect Dis 2001; 41: 171-5.

6. Livermore DM. Bacterial resistance: origins, epidemiology, and impact. Clin Infect Dis 2003; 36 (Suppl 1): S11-23.

7. McGowan JE, Tenover FC. Confronting bacterial resistance in healthcare settings: a crucial role for microbiologists. Nat Rev Microbiol 2004; 2: 251-8.

8. Morens DM, Folkers GK, Fauci AS. The challenge of emerging and re-emerging infectious disease. 2004, Nature vol. 430.

9. National Committee for Clinical Laboratory Standards. Methods for Dilution Antimicrobial susceptibility tests for bacteria that grow aerobically; Approved StandardSixth Edition: M7-A6 and supplement M100-S14, Wayne, PA, 2003.

10. Nicolau D. Clinical and economic implications of antimicrobial resistance for the management of community-acquired respiratory tract infections. J Antimicrob Chemother 2002; 50 (Suppl S1): 61-70.
11. O'Brien TF. Emergence, spread and environmental effect of antimicrobial resistance: how use of an antimicrobial anywhere can increase resistance to any antimicrobial anywhere else. Clin Infect Dis 2002; 34 (Suppl 3): s78-84.

12. Raoult D, Fournier PE, Drancourt M. What does the future hold for clinical microbiology. Nat Rev Microbiol 2004; 2: 151-9.

13. Tenover FC. Development and spread of bacterial resistance to antimicrobial agents: an overview. Clin Infect Dis 2001; 33 (Suppl 3): S108-S115.

14. Wood MJ, Moellering RC. Microbial resistance: bacteria and more. Clin Infect Dis 2003; 36 (Suppl 1): S2-3.

\section{Eugenio A. Debbia}

Università degli Studi di Genova

DISCAT - Sez. Microbiologia

Largo Rosanna Benzi 10

16132 Genova - Italia

$\mathrm{Tel}++$ 010-3537655 - Fax ++ 0103537698

E-mail: eugenio.debbia@unige.it 